\title{
Autoimmune polyendocrinopathy type 1
}

INSERM

\section{Source}

INSERM. (1999). Orphanet: an online rare disease and orphan drug data base.

Autoimmune polyendocrinopathy type 1. ORPHA:3453

Autoimmune polyendocrinopathy type 1, or APECED syndrome, is a genetic disease that manifests in childhood or early adolescence with a combination of chronic mucocutaneous candidiasis, hypoparathyroidism and autoimmune adrenal failure. 